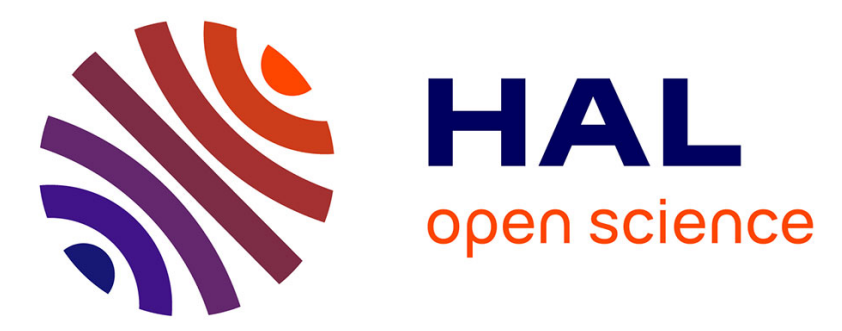

\title{
Holographic microscopy and diffractive microtomography of transparent samples
}

Bertrand Simon, Matthieu Debailleul, Vincent Georges, Olivier Haeberlé, Vincent Lauer

\section{- To cite this version:}

Bertrand Simon, Matthieu Debailleul, Vincent Georges, Olivier Haeberlé, Vincent Lauer. Holographic microscopy and diffractive microtomography of transparent samples. Measurement Science and Technology, 2008, 19, pp.074009. 10.1088/0957-0233/19/7/074009 . hal-00869405

\section{HAL Id: hal-00869405 https://hal.science/hal-00869405}

Submitted on 2 Dec 2013

HAL is a multi-disciplinary open access archive for the deposit and dissemination of scientific research documents, whether they are published or not. The documents may come from teaching and research institutions in France or abroad, or from public or private research centers.
L'archive ouverte pluridisciplinaire HAL, est destinée au dépôt et à la diffusion de documents scientifiques de niveau recherche, publiés ou non, émanant des établissements d'enseignement et de recherche français ou étrangers, des laboratoires publics ou privés. 


\title{
Holographic microscopy and diffractive microtomography of transparent samples
}

\author{
Bertrand Simon $^{1}$, Matthieu Debailleul ${ }^{1}$, Vincent Georges ${ }^{1}$, \\ Olivier Haeberlé ${ }^{1}$, Vincent Lauer $^{2}$ \\ ${ }^{1}$ Laboratoire MIPS, IUT Mulhouse, 61 rue A. Camus 68093 Mulhouse Cedex \\ France \\ ${ }^{2}$ Lauer Microscopie, 1bis Rue des Blés, 68200 Mulhouse France \\ E-mail: lauer@waika9.com, olivier.haeberle@uha.fr
}

\begin{abstract}
We present an optical tomographic diffractive microscope, a device able to image a complex refractive index distribution in three dimensions. Theoretical foundations are first recalled: diffraction under the first Born approximation explains the link between diffracted beam, object frequencies and physical properties of the object. We then describe our experimental setup, recording 2-D interferograms in the image space, and detail the image reconstruction process underlying our tomographic microscope, which involves 2$\mathrm{D}$ transforms of the recorded interferograms, a peculiar 3-D mapping of the data, and a final 3-D Fourier reconstruction. We apply tomographic reconstruction to diatom skeletons, unicellular algae with cell walls made of silica, and compare it to holographic reconstruction. We further apply it to pollen grains and show differences between the real and imaginary parts of the measured complex refractive index. Finally, we also recall alternative tomographic methods.
\end{abstract}

PACS numbers: 42.30.Wb, 42.30.Kq, $42.40 \mathrm{Kw}$

Image reconstruction, tomography, Fourier optics, holographic interferometry, other holographic techniques. Submitted to: Meas. Sci. Technol. 


\section{Introduction}

Optical tomographic microscopy allows for three-dimensional imaging of unstained samples by using successive illuminations in various conditions, recording of the diffracted waves, and finally reconstructing the sample from the diffracted waves. In this paper we use the approach developed in [1] in which the sample is fixed and the direction of a coherent illuminating wave is varied. This method allows for high-resolution three-dimensional imaging and has a potential for high-speed, threedimensional imaging, as the imaging can be performed without moving the sample nor moving any heavy component: the direction of the illuminating beam can be varied using a lightweight galvanometer mirror or even an acousto-optic deflector. In our setup, we use a detector placed in an image plane, which simplifies the imaging device and improves speed.

In the present paper, we first describe the general principles underlying the obtainable resolution. We describe our optical setup and the method for obtaining three-dimensional images. We also discuss existing alternatives to do optical tomographic microscopy.

\section{Theoretical background}

We first give a short description, based on the approach given by Wolf [2], within the first Born approximation for weakly scattering objects. We recall the scalar propagation equation in an inhomogeneous medium, neglecting polarization effects:

$$
\left(\nabla^{2}+k(\mathbf{r})^{2}\right) \psi(\mathbf{r})=0 \quad \text { with } k(\mathbf{r})=\frac{2 \pi}{\lambda} n(\mathbf{r})
$$

wherein $n(\mathbf{r})=\sqrt{\frac{\mu(\mathbf{r}) \epsilon(\mathbf{r})}{\mu_{0} \epsilon_{0}}}$ is the complex refractive index and $\lambda$ is the wavelength in vacuum of an incident plane wave.

Such an equation cannot be solved directly. Writing a solution in terms of Green's function

$$
G(\mathbf{r})=\frac{\exp \left(j k_{i}|\mathbf{r}|\right)}{4 \pi|\mathbf{r}|}
$$

wherein $k_{i}=2 \pi \frac{n_{0}}{\lambda}$ is the modulus of the wave vector of the incident plane wave in the immersion medium having index $n_{0}$, leads to [3]:

$$
\psi_{s}(\mathbf{r})=\int G\left(\mathbf{r}-\mathbf{r}^{\prime}\right) O\left(\mathbf{r}^{\prime}\right) \psi\left(\mathbf{r}^{\prime}\right) \mathrm{d} \mathbf{r}^{\prime}
$$

where $\psi(\mathbf{r})=\psi_{s}(\mathbf{r})+\psi_{i}(\mathbf{r})$ is the total field, $\psi_{i}(\mathbf{r})$ being the illumination field and $\psi_{s}(\mathbf{r})$ the scattered field, and

$$
O(\mathbf{r})=k_{i}^{2}\left(\frac{n^{2}(\mathbf{r})-n_{0}^{2}}{n_{0}^{2}}\right)
$$

is the object function, $n$ being the index of the specimen. The Born approximation assumes that objects are weakly scattering only. Then the diffracted field is small compared to the incident field and equation 3 is rewritten as:

$$
\psi_{s}(\mathbf{r})=\int G\left(\mathbf{r}-\mathbf{r}^{\prime}\right) O\left(\mathbf{r}^{\prime}\right) \psi_{i}\left(\mathbf{r}^{\prime}\right) \mathrm{d} \mathbf{r}^{\prime}
$$


This equation can be interpreted as follows: Green's function represents an outgoing scalar field scattered by a point object. The field scattered by a point is thus the product of the Green function by the value at that specific point of the object function and the value at that specific point of the incident scalar field. The field scattered by the entire object is obtained by integration on all points of the object, i.e. it is obtained by convolution of Green's function by the product of the object function and the incident scalar field. Adding an ingoing scalar field to Green's function will not modify the detected field since in the far-field only the outgoing field is detected due to the orientation of detectors. It will modify the calculated field scattered by the entire object, but not the part of that field which we detect. This method, which is a scalar equivalent of the vector method used in [1], allows us to write modified equations between the object function and the field as detected, which involve only homogeneous propagating waves. By adding a suitable ingoing field to the Green function (eq.2), we obtain :

$$
G_{m}(\mathbf{r})=\frac{\exp \left(j k_{i}|\mathbf{r}|\right)}{4 \pi|\mathbf{r}|}-\frac{\exp \left(-j k_{i}|\mathbf{r}|\right)}{4 \pi|\mathbf{r}|}
$$

the Fourier transform of $G_{m}(\mathbf{r})$ is:

$$
\tilde{G}_{m}\left(\mathbf{k}_{\mathbf{d}}\right)=\frac{j}{4 \pi k_{i}} \delta\left(\left|\mathbf{k}_{\mathbf{d}}\right|-k_{i}\right)
$$

i.e. it represents a purely monochromatic propagative scalar field, unlike the Green function itself.

Thus in equation ( 5$)$ :

- we replace Green's function $G(\mathbf{r})$ by the modified Green function $G_{m}(\mathbf{r})$.

- we consider an incident plane wave $\psi_{i}(r)=a_{i} \exp \left(j \phi_{i}\right) \exp \left(j \mathbf{k}_{\mathbf{i}} \cdot \mathbf{r}\right)$.

- we obtain a modified scattered field $\psi_{m}(r)$ which replaces the scattered field $\psi_{s}(r)$ and cannot be distinguished from the scattered field by detectors placed in the far-field and looking towards the object. This modified field can be viewed thought of as the detected field. It is also the field which would be reconstructed by a backscattering algorithm since such algorithms assume a monochromatic homogeneous scalar field.

In the Fourier space equation 5 then becomes:

$$
\tilde{\psi}_{m}\left(\mathbf{k}_{\mathbf{d}}\right)=\frac{j}{4 \pi k_{i}} a_{i} \exp \left(j \phi_{i}\right) \delta\left(\left|\mathbf{k}_{\mathbf{d}}\right|-\left|\mathbf{k}_{\mathbf{i}}\right|\right) \tilde{\mathbf{O}}\left(\mathbf{k}_{\mathbf{d}}-\mathbf{k}_{\mathbf{i}}\right)
$$

From 8, we then obtain the complex amplitude of the scalar field in the Fourier space:

$$
A\left(\mathbf{k}_{\mathbf{d}}\right)=\frac{j}{4 \pi k_{i}} a_{i} \exp \left(j \phi_{i}\right) \tilde{O}\left(\mathbf{k}_{\mathbf{d}}-\mathbf{k}_{\mathbf{i}}\right)
$$

wherein this complex amplitude $A\left(\mathbf{k}_{\mathbf{d}}\right)$ depends only on the direction of $\mathbf{k}_{\mathbf{d}}$ and is defined by :

$$
\tilde{\psi}_{m}\left(\mathbf{k}_{\mathbf{d}}\right)=A\left(\mathbf{k}_{\mathbf{d}}\right) \delta\left(\left|\mathbf{k}_{\mathbf{d}}\right|-\left|\mathbf{k}_{\mathbf{i}}\right|\right)
$$

Equation 9 links the complex amplitude of the measured scattered field to the object function via their Fourier transforms. In the 3D tomographic microscope a detector placed in a Fourier plane detects the complex amplitude $A\left(\mathbf{k}_{\mathbf{d}}\right)$. This complex amplitude is then corrected for variations of $\phi_{i}$ and $a_{i}$ and mapped onto the object function $\tilde{O}\left(\mathbf{k}_{\mathbf{o}}\right)$. 
This scalar approximation has been shown to give good results and we will use it in the following. A more elaborate version can be obtained by replacing Green functions by the electric field vector radiated by a dipole [1].

Also, note that under the first Born approximation, the reconstruction is strictly valid for a low phase change only, which is proportional to both the object index and size, while under the Rytov approximation, "the size of the object is not a factor" (from [3]) and therefore the Rytov approximation is expected to give better results for larger objects.

We will use the spatial frequencies $\mathbf{f}_{\mathbf{d}}=\mathbf{k}_{\mathbf{d}} / \mathbf{2} \pi$ (spatial frequency of the diffracted wave) $\mathbf{f}_{\mathbf{i}}=\mathbf{k}_{\mathbf{i}} / \mathbf{2} \pi$ ( spatial frequency of the illumination wave) and $\mathbf{f}_{\mathbf{0}}=\mathbf{k}_{\mathbf{0}} / \mathbf{2} \pi$ (spatial frequency of the object). Using equation (9) we find that spatial frequencies are linked by:

$$
f_{d}=f_{i}+f_{o}
$$
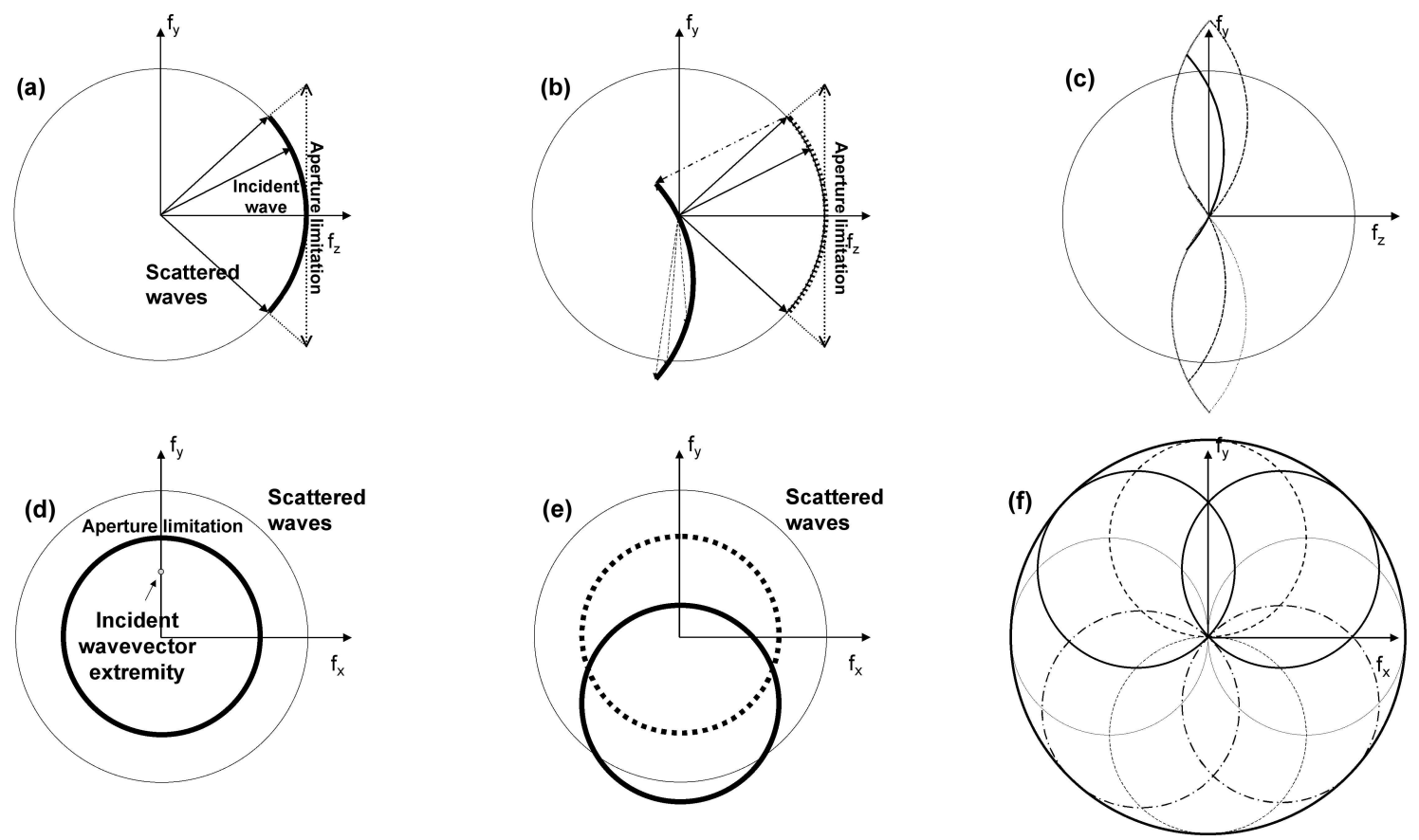

Figure 1. Principle of diffractive tomographic microscopy: construction of the detected frequency support in the case of a transmission microscope.

When considering a transmission geometry and only one direction of illumination, only one half of the Ewald sphere could be recorded at best. Moreover this half sphere is restricted to a cap of sphere only, because of the limited numerical aperture of the microscope objective $\left(\mathrm{NA}_{o b j}\right)$ used in the detection system.

The wave diffracted by the weakly scattering object is recorded in both amplitude and phase using a holographic recording setup. As shown on figure 1(a), a very limited subset of the diffracted frequencies can be measured. For a given incident direction $\mathbf{f}_{\mathbf{i}}$, and an observation direction $\mathbf{f}_{\mathrm{d}}$, the corresponding spatial frequency of the object permittivity is $\mathbf{f}_{\mathbf{d}}-\mathbf{f}_{\mathbf{i}}$. The set of spatial frequencies $\mathbf{f}_{\mathbf{d}}-\mathbf{f}_{\mathbf{i}}$ which are recorded is 
shown as a bold arc of circle on figure $1(\mathrm{~b})\left(-\mathbf{f}_{\mathbf{i}}\right.$ is indicated by the dashed arrow on figure 1(b)). The resulting limited subset of recorded object frequencies will lead to low imaging quality, as will be shown in section 5 .

The recorded spatial frequency space can be filled using successive variable directions of illumination, leading to recording a more complete subset of the threedimensional frequency representation of the object, in order to perform a more accurate reconstruction of the object.

In our approach, the detection system and the specimen are fixed, and tomographic acquisition is performed by changing the angle of incidence of the illumination wave. The incident direction is changed but the observation directions are the same. After proper shifting with respect to the incident illumination, other sets of the object frequencies are detected as shown in figure 1(c). Using a tilted illuminating wave permits to record higher frequencies from the Fourier transform of the specimen. It is therefore desirable to use high illumination angles. In our setup, this is performed using a high NA condenser, as in a classical incoherent transmission microscope.

The process depicted on figure $1(\mathrm{a}, \mathrm{b}, \mathrm{c})$ in the $\left(f_{y}, f_{z}\right)$ plane is also shown on figure $1(\mathrm{~d}, \mathrm{e}, \mathrm{f})$ in the $\left(f_{x}, f_{y}\right)$ plane. The different parts of figure 1 give a complete explanation of how the spatial frequencies space is filled in a transmission setups. For example, the upper dashed disk in figure $1(\mathrm{f})$ corresponds to an illumination at $90^{\circ}$ azimuth angle. It shows up as the upper dashed arc of circle in figure 1(c).

When a large number of incidences is used, the support of the detected frequencies in the $(x-y)$ plane becomes a disk as shown in figure $1(\mathrm{f})$.

In the $(y-z)$ plane, the Fourier domain scanned by the diffractive tomographic microscope when a large number of incident angles is used takes the form of the well-known butterfly shaped support of the Optical Transfer Function (OTF) for a transmission microscope.

\section{Experimental setup}

Figure 2(a) depicts a sketch of the experimental setup of the tomographic diffractive microscope as originally described in [1]. It is based on a Mach-Zehnder setup used to realise phase-shifting holography [5]

The light issued from a He-Ne laser is separated into a reference wave and an illumination wave by the beam splitter BS1. The reference wave is made plane using lenses L3 and L4. In order to perform phase-shifting holography, its phase is controlled by a mirror mounted on a piezoelectric element. Lenses L1 and L2 are used to illuminate the specimen with a plane illumination wave. The direction of this illumination wave is controlled via the motorized tilting mirror and the condenser: a slight tilt of the mirror generates a large angular variation of the plane wave illuminating the specimen. After diffraction by the specimen, the diffracted wave is collected by the objective. It then passes through lenses LT and L5 and reaches the CCD sensor which is placed at the focal point of the non-diffracted part of the illuminating wave, that is in a Fourier plane.

The reference wave is recombined with the diffracted wave using BS2. The interferences obtained are recorded on the CCD sensor. For each direction of the illumination wave, four images are recorded on the CCD with the phase of the reference wave being shifted by $\pi / 2$ between each image. A complex image is computed using 
a)

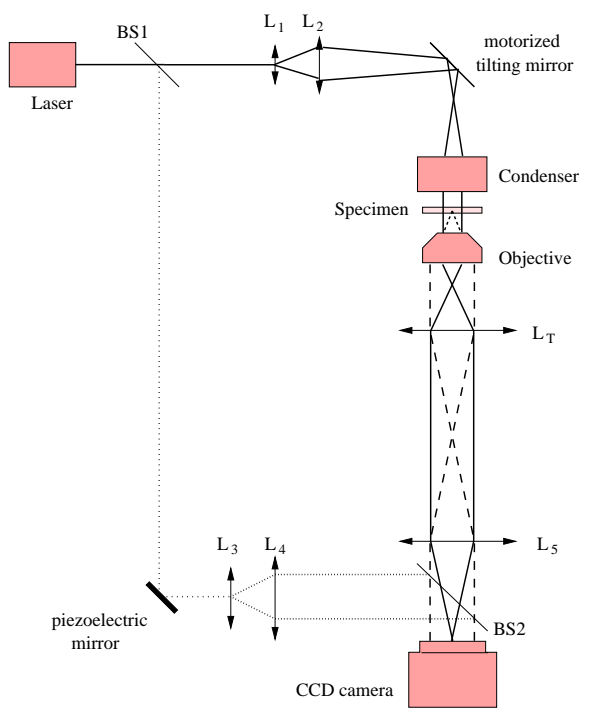

b)

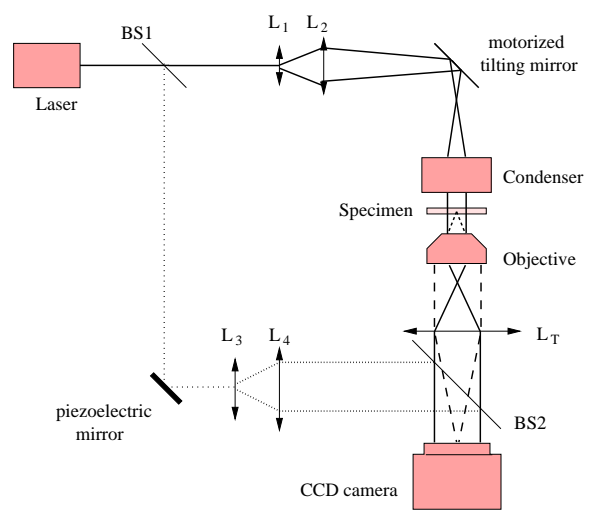

Figure 2. Experimental setup of the transmission diffractive tomographic microscope. In 2(a) the CCD plane is localised in a Fourier plane, whereas in figure 2(b) it is localised in an image plane.

the formula $A=I_{0}-I_{\pi}+j\left(I_{\pi / 2}-I_{3 \pi / 2}\right)$ where $A$ is the complex value, defined for each pixel of the CCD.

However, placing the CCD sensor in a Fourier plane has a major drawback: in such a plane, the non-diffracted part of the illuminating wave generates a very bright spot on the camera. If this bright spot is accurately detected, the diffracted wave itself is usually below the detection threshold of the sensor.

We solved this problem by placing the CCD sensor in an image plane as presented in 2(b). In this case, the non-diffracted part of the illuminating wave generates a continuous background, which does not tend to saturate the sensor. After the phaseshifting step, a numerical two dimensional Fourier transform is performed on the complex image, yielding the same spectrum which would have been directly obtained if the camera had been placed in a Fourier plane.

Note that changing the direction of illumination may be limited by total internal reflection. For living biological specimens, which index of refraction may be approximated to that of water (1.33), using an oil immersion condenser with numerical aperture N.A.=1.4 will lead to total internal reflection for illumination at the glass-toward-water interface. Similarly, the collection of the diffracted wave by the microscope objective would also be limited to N.A.=1.33 (at best). In order to avoid this phenomenon, and as the samples we studied here are non-living diatom skeletons and environment resistant pollens, we actually embedded these specimens into the same immersion oil as the objective or the condenser. Doing so, we could check that the illumination wave (without specimen) is indeed collected by the objective even at maximum illumination angle, which ensures that both the illumination and the dectection are performed at maximal numerical aperture. For 3-D imaging of living cells, using water immersion objectives (with N.A.=1.2) would probably be more appropriate. 


\section{Normalisation before mapping of the data}

Equation 11 shows that one must properly determine $\mathbf{f}_{\mathbf{i}}$ in order to remap the Fourier space as described by figure 1. Because the object is weakly scattering, the location of this single frequency corresponds to the location of the maximum of intensity of the diffracted wave. We determine $\mathbf{f}_{\mathbf{i}}$ by extracting the coordinates $\mathbf{f}_{\mathrm{dmax}}=\mathbf{f}_{\mathbf{i}}$ of the maximum of intensity of the diffracted wave (figure 3(a)). The complex amplitude $A_{d}\left(\mathbf{f}_{\mathbf{d m a x}}\right)$ at that point is equal to the complex amplitude of the illumination wave $a_{i} \exp \left(j \phi_{i}\right)$. Because of mechanical limitations, the illumination wave may be affected by random phase shifts $\phi_{i}$ (because mechanical rotation is inevitably accompanied with parasitic translations) as well as possible vibrations. Furthermore laser intensity fluctuations may happen and also the transmission of the illumination and detection optics may vary with the angle of incidence, changing $a_{i}$. One must compensate for these possible fluctuations. This is simply performed by dividing the amplitude of the diffracted wave by the complex amplitude of the illumination wave (figure 3(b)):

$$
A_{\text {norm }}\left(\mathbf{f}_{\mathbf{d}}\right)=A_{d}\left(\mathbf{f}_{\mathbf{d}}\right) / A_{d}\left(\mathbf{f}_{\mathbf{d} \max }\right)
$$

In application of equation 11 the normalised complex amplitude is then translated as shown on figure $3(\mathrm{c})$, which yields the complex amplitude of the object Fourier transform $A_{o b j}$ :

$$
A_{o b j}\left(\mathbf{f}_{\mathbf{o}}\right)=A_{\text {norm }}\left(\mathbf{f}_{\mathbf{d}}-\mathbf{f}_{\mathbf{d m a x}}\right)
$$

Object spatial frequencies mapping

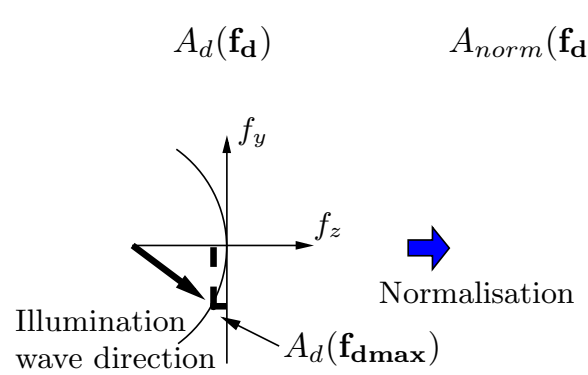

(a)

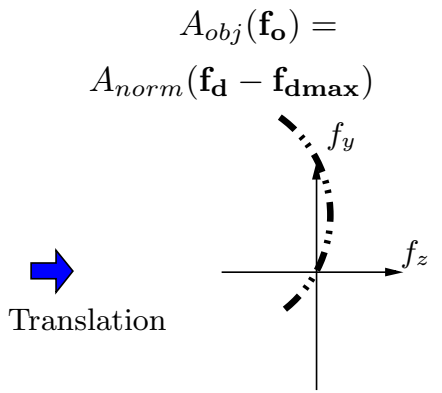

(c)

Figure 3. Normalisation for mapping of the Fourier frequencies

These normalisations must be repeated for the successive diffracted wavefronts obtained by varying the illumination direction. Note that this step constitutes a vital point in practice for a correct specimen reconstruction. Omitting this correction would lead to randomization of the acquired data that may result in a totally unexploitable image.

The successive complex amplitudes are accumulated in the Fourier space, yielding the frequency representation of the object. Some redundant frequencies may appear; in this case, we take the average of the complex amplitudes located at this redundant frequencies, which allows for an important reduction of the noise. Finally, performing a three-dimensional inverse Fourier transform yields the object three-dimensional representation. 


\section{Results}

A comparison between holographic (1 illumination angle) and tomographic reconstructions (1000 angles) is shown on figure 4 for a $(y-z)$ and two $(x-y)$ planes. We imaged a Coscinusdiscus sp diatom whose skeleton (or frustule) is made of silica. These data have been acquired with $\mathrm{NA}_{o b j}=1.4$ and in the tomography case, with a condenser numerical aperture corresponding to $\mathrm{NA}_{\text {cond }}=1.4$.

The vertical slicing capabilities of optical tomography are well illustrated by figure 4. In the left column of figure 4, the hologram is disturbed by out of focus parts of the object and thus does not allow for reasonable image quality. In the right column of figure 4 , the thin slicing capabilities of optical tomography allows the obtention of a vertical slice (figure 4(b)) and of a middle (figure 4(d)) and top (figure 4(f)). As can be seen on figure (figure 4(f)), the top slice shows only the very top of the diatom and is undisturbed by out of focus information.

Diffractive tomographic microscope under the first Born approximation has the capability to image simultaneously the refractive index and the absorption of a semitransparent object. On figure (5), both refractive index and absorptivity (imaginary part of the complex refractive index) of a snowdrop pollen are presented in a $(x-y)$ plane at different $z$ values. These two informations show different structures inside the pollen (figure 5(a) and (b)). The absorptive part of an organelle (white arrows) is well contrasted on figure 5(d) whereas almost invisible on figure 5(c). On the other hand, the refractive index on figure $5(\mathrm{e})$ is better contrasted than absorptivity on figure 5 (f).

Our computation yields to the object function of equation 4 (or scattering potential). For small $n(\mathbf{r})-n_{0}$ this object function is simplified as:

$$
O(\mathbf{r})=2 \frac{k_{i}^{2}}{n_{0}}\left(n(\mathbf{r})-n_{0}\right)
$$

so that the object function is proportional to the complex refractive index, i.e. its real part is proportional to the real part of the refractive index and its imaginary part is proportional to the absorptivity.

Nevertheless, it should be noticed that the actual experimental setup has some drawbacks : as seen on figure 4(d), horizontal walls are difficult to image, due to the missing cone along the optical longitudinal axis in the Fourier space.

\section{Discussion}

Tomographic microscopy has become the subject of intensive research and a number of alternative techniques have been developed. Different methods have been proposed and used for solving the problem of properly filling a reasonable part of the frequency space, mapping the data into the frequency space, detecting the diffracted wave, and improving the resolution.

A common feature to transmission microscopes is the presence of the so-called missing cone in the frequency space, which strongly limits the resolution along the optical axis. A popular alternative to obtain a more isotropic resolution is to rotate the sample instead of varying the illumination direction $[7,8,9]$. In such a setup, the sample must be prepared within a rotating cylinder, which maybe more difficult. This setup can also be modified for using non-coherent illumination [11].

Another alternative is to use simultaneous illumination by more than one parallel beam [6, 12]. However, only a limited number of different illumination directions can 

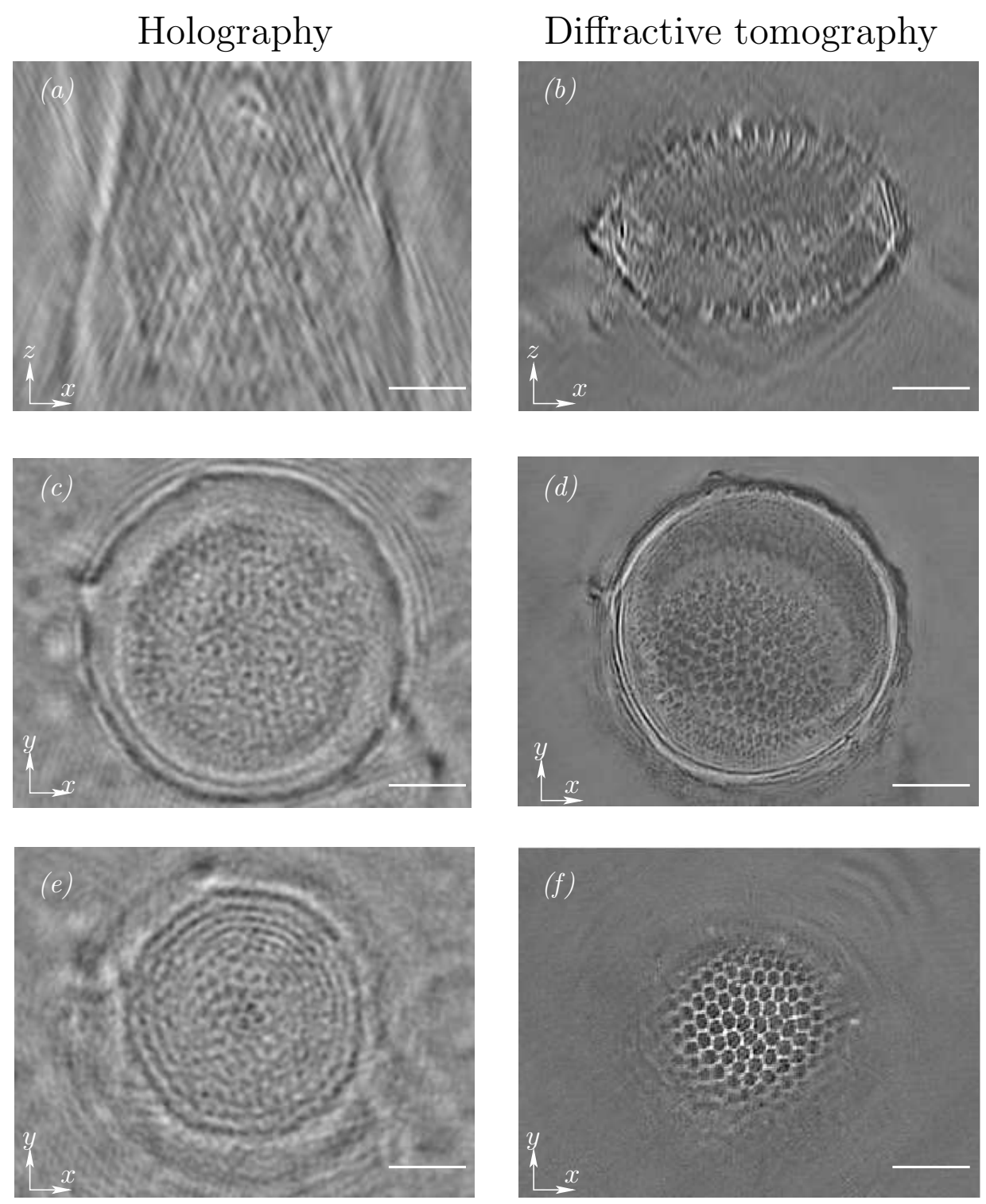

Figure 4. Holography vs tomography. On the left side, three slices extracted from the holographic reconstruction are shown : figure (4a) shows a $(x-z)$ plane and figures $4(\mathrm{c}, \mathrm{e})$ a $(x-y)$ plane. On the right side, the same object is shown in the tomographic case (1000 angles): figure 4(b) shows a $(x-z)$ plane and figure $4(\mathrm{~d}, \mathrm{f})$ show a $(x-y)$ plane corresponding to figure $4(\mathrm{c}, \mathrm{e})$. Scale bar represents 5 $\mu \mathrm{m}$. 

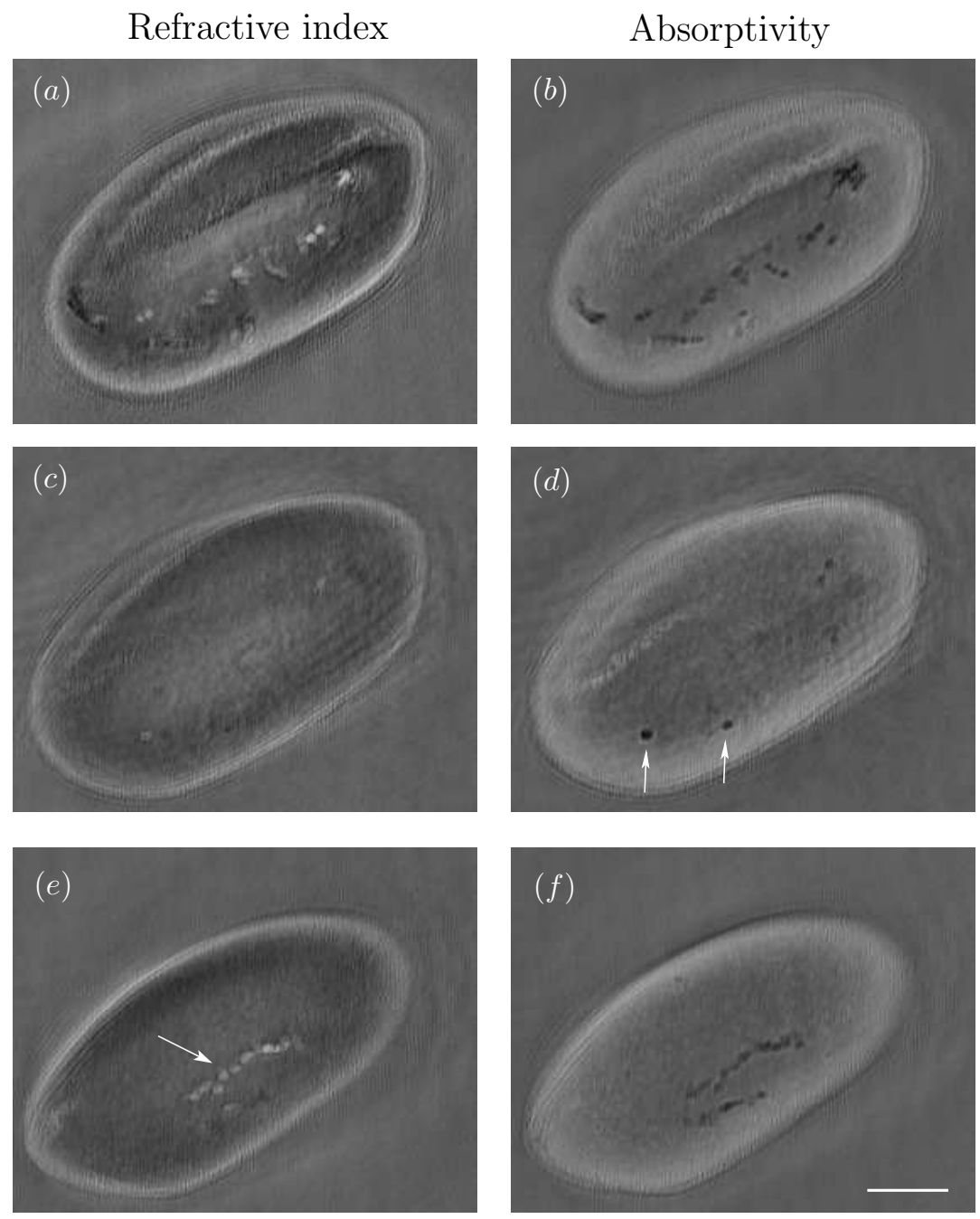

Figure 5. Refractive index (left side) and absorption (right side) parts of a snowdrop pollen for different $z$ planes.

be used in this method, the advantage being that they could in principle be used simultaneously, which would improve the acquisition speed.

Varying the wavelength [12] can also improve the imaging capabilities because it allows for the acquisition of different information, as the curvature radius of the recorded cap of spheres changes with the wavelength (a missing-cone does remain). For 2-D imaging only, the lateral resolution is anyway determined by the shortest wavelength, but for 3-D imaging, a more complete subset would be recorded, which would improve the imaging capabilities along the optical axis.

The possibility of simultaneously using reflexion and transmission to improve the frequency support was proposed in $[1,19]$ but has not yet been reduced to practice.

The method used in the present paper for filling the frequency space is to sequentially vary the directions of the illumination beam. It has the advantage of 
yielding an easy to use instrument without any unusual sample preparation, and yet allowing a high number of illumination to be used and a reasonably large part of the frequency space to be filled. However, each of the above alternatives has specific advantages in certain situations; especially, each method allows for a different filling of the frequency space.

We used phase stepping holography to measure the diffracted field. A possible alternative is to use in-line holography [14] rather than using a separate illumination and reference beam. Doing so, only one hologram is to be recorded and processed in order to measure the diffracted components. This would also help to increase the acquisition speed. However the phase retrieval algorithms which must be used in this case cannot easily recover both the phase and amplitude of the detected diffracted beam. In-line holography can be modified for non-coherent light [15]. In this case, the sample must be assumed to be a purely dephasing one, i.e. non-absorptive. This hypothesis may often be fulfilled in pratice, but must be handled with care: it is for example clearly taken at fault for pollens, as shown by figure (5). Alternatively, offaxis holography also permits to record the diffracted field with only one hologram[8]. In that case, a camera with a larger CCD chip should be used in order to record the same field of view with the same resolution [5].

In this work, the mapping of the frequency space is realised using the first Born approximation, and takes into account the diffraction in order to reach a subwavelength resolution. Such approximation has already been employed in [7] and [1]. In $[8,9,11,18]$, mapping is performed using a path integrated phase assumption and thus the reconstruction is realised using backprojection algorithm (Radon transform) and consequently may not be strictly considered as diffraction tomography. In $[9,10]$, a comparison between the Radon transform and exact mapping is detailed. In particular, the authors have highlighted possible reconstruction artefacts when using Radon reconstruction. When trying to get the maximum resolution, diffraction has to be taken into account in the reconstruction process.

In order to even further improve the resolution of the images, a priori informations about the observed object can be used for the reconstruction [16, 17]. Also, non-linear reconstruction methods can improve the quality of the images. These techniques could become a useful complement to the usual, linear version of optical tomography detailed in this paper, but their use still has to be investigated for such a set-up. Note also that the Fourier method we used requires that the Born approximation is satisfied, namely that the sample both is weakly absorbing and induces small phase changes only. The Rytov approximation, which is also linear, would be expected to provide better results for larger objects. Therefore, the method for microtomographic images reconstruction probably has to be tweeked with respect to the considered specimen and experimental goals, trying to detect the tiniest features in a smaller specimen, or trying to image a much larger specimen with a more modest resolution.

An important point in practice for biological application is also the speed of acquisition and reconstruction of the 3-D images. Our set-up is presently slow. For 1000 angles of illumination, 4000 holograms have to be recorded. The piezoelectric element has to physically translate a mirror, and the stepper motors driven-, tip-tilt mirror used for controlling the illumination must both ensure vibrationless motions. We therefore are restricted to slow movements, and have introduced temporisations to wait for vibration damping. As a consequence, 4000 holograms take 40 minutes to be recorded. This obviously is incompatible with live-cell imaging, but is tolerable for our specimens, or even for fixed cells. 
Replacing these elements by high-speed systems may permit to overcome these limitations as demonstrated by Choi et al.[18], who recently presented a high speed version of our set-up. The reconstruction method they used being however based on an inverse Radon transform, the accuracy of the reconstruction is lower, as shown for example by Gorski and Olsten [9]. We now plan to improve the speed of acquisition of our set-up, by replacing the speed limiting mechanical elements by faster systems, as for example galvanometric mirrors instead of a stepper motor driven mirror for illumination control, an electro-optic modulator instead of a piezo mirror for phase stepping, and a high-speed camera.

The reconstruction is fast: for a 32 bits 512 voxel complex final image, the 1000 2-D transforms, the 3-D mapping of the data and the final 3-D Fourier transform take less than 5 minutes on a 2.6Ghz dual-core PC using 64 bits Linux, and fast 3-D FFT transforms [20]. Note that most of the computational time is spent on reading the data on the hard-disk and not in the Fourier transforms themselves. Presently, we perform all the computations after completing the acquisitions. Alternatively, one could also perform all the 2-D computations and 3-D mapping progressively with the acquisitions, so that only the final 3-D Fourier transform would remain, which would also permit to save time.

Note that our method is comparable to the above described alternate methods in terms of reconstruction speed, as the involved mathematical operations are the same for all methods. However, rotating the specimen for tomography presents the drawback that inevitable parasitic mechanical translations of the sample oblige to a precise numerical compensation before mapping the information in the $3-\mathrm{D}$ Fourier space [10]. While these parasitic movements also happens when tilting the mirror in our set-up, their corrections is very easy, as explained in section 4 (Fig. 4). This probably explains that results already presented by various authors using specimen rotation do not present optimal resolution, because then an inverse Radon transformation seems easier. Also, biologist often tend to prefer preparing their sample using more classical glass slide with cover glass method instead of having to insert it within a microcapillary.

\section{Conclusions}

Tomographic microscopy allows for quantitative three-dimensional imaging with optimized resolution. Whereas fluorescence microscopy has experienced a strong evolution with the generalized use of confocal fluorescence microscopy, there has been no corresponding evolution in transmission or reflexion microscopy. Use of confocal microscopes in transmission has always proven difficult and did not lend itself to a generalized use. We hope that tomographic microscopy will bring to transmission microscopy the improvement, which it needs to keep pace with confocal fluorescence imaging. The tomographic microscopy method presented in this paper should allow high-resolution and high-speed three-dimensional observation of unstained samples. Furthermore, it may allow confocal images to be superimposed to quantitative threedimensional images, instead of the more usual DIC contrast images.

\section{Acknowledgements}

We gratefully acknowledge Dominique Voisin for the diatoms preparation and Joël Lambert for valuable help in the design and construction of the microscope. 


\section{References}

[1] Lauer V 2002 New approach to optical diffraction tomography yielding a vector equation of diffraction tomography and a novel tomographic microscope, Journal of Microscopy, 205, 165

[2] Wolf E 1969 Three-dimensional structure determination of semi transparent objects from holographic data Optics Communications 1153

[3] Slaney M, Kak A C, Larsen L E 1984 Limitations of Imaging with First-Order Diffraction Tomography IEEE transactions on microwaves theory and techniques $\mathbf{3 2} 860$

[4] Born M, Wolf E 1991 Principles of Optics: Electromagnetic Theory of Propagation, Interference and Diffraction of Light. Principles of Optics: Electromagnetic Theory of Propagation, Interference and Diffraction of Light

[5] Zhang T Yamaguchi I 1997 Phase-shifting digital holography, Optics Letters 221268

[6] Fercher AF et al 1979 Image formation by inversion of scattered field data : experiments and computational simulations Applied Optics 182427

[7] Woodford P, Turpin T, Rubin M, Lapides J, Price C 1996 The synthetic aperture microscope, experimental results SPIE Proceedings 2751230

[8] Charrière F, Pavillon P, Colomb T, Marquet P, Rappaz B, Depeursinge C, Heger J T, Mitchell A D 2006 Living specimen tomography by digital holographic microscopy : morphometry of testae amoeba Optics Express 147005

[9] Gorski W, Osten W 2007 Tomographic imaging of photonic crystal fibers Optics Letters 321977

[10] Vertu S, Yamada I, Delaunay J-J and Haeberlé O 2008 Tomographic observation of transparent objects under coherent illumination and reconstruction by filtered backprojection and Fourier diffraction theorem SPIE Proceedings $\mathbf{6 8 6 1} 686103$

[11] Fauver M and Seibel E J 2005 Three-dimensional imaging of single isolated cell nuclei using optical projection tomography Optics Express 134210

[12] Mico V, Zalevsky Z, Garcia J 2007 Synthetic aperture microscopy using off-axis illumination and polarization coding Optics Communications 276209

[13] Kühn J et al 2007, Real-time dual-wavelength digital holographic microscope with a single hologram acquisition Optic Express 157231

[14] Devaney AJ, Schatzberg A 1992 The coherent optical tomographic microscope SPIE Proceedings $\mathbf{1 7 6 7} 62$

[15] Noda T, Kawata S, Minami S 1992 Three-dimensional phase-contrast imaging by a computedtomography microscope Applied Optics 31670

[16] Kawata S, Nakamura O, Minami S 1987 Optical microscope tomography I Support constraints Journal of the Optical Society of America A 4392

[17] Belkebir K, Chaumet PC, Sentenac A. 2006 Influence of multiple scattering on three-dimensional imaging with optical diffraction tomography. J Opt Soc Am A Opt Image Sci Vis. 23586

[18] Choi W et al. 2007 Tomographic phase microscopy Nature vol.4 717

[19] Fukutake N, Milster T D, 2007 Proposal of three-dimensional phase contrast holographic microscopy Optics Express, 15, 12662

[20] M. Frigo, S.G. Johnson, 2005 The Design and Implementation of FFTW3 Proceedings of the IEEE 932216. 